\title{
Síntomas de angina en la mujer colombiana: una metasíntesis
}

\author{
Fanny Rincón-Osorio*, Luz P. Díaz-Heredia, Elizabeth Vargas-Rosero, \\ Renata V. González-Consuegra, Luisa F. Moscoso-Loaiza, \\ Josefina Manosalva-Murillo, Viviana M. Céspedes-Cuevas y Magda L. Flórez-Flórez
}

Facultad de Enfermería, Grupo de investigación Cuidado para la salud Cardiorrespiratoria, Universidad Nacional de Colombia, Sede Bogotá, Bogotá, Colombia

Recibido el 19 de octubre de 2017; aceptado el 15 de diciembre de 2017

Disponible en Internet el 12 de enero de 2018

\author{
PALABRAS CLAVE \\ Angina pectoris; \\ Mujer; \\ Investigación \\ cualitativa
}

\begin{abstract}
Resumen
Objetivo: conocer y puntualizar los atributos de los síntomas de angina expresados por la mujer colombiana.

Método: estudio metasintético de nueve reportes de investigación con abordaje cualitativo desarrollados entre los años 2006 y 2012. La investigación se condujo con base en la propuesta metodológica de Noblit y Hare y la teoría de los síntomas desagradables de Lenz et al.

Resultados: los atributos del síntoma evidenciaron que inicia de manera insidiosa y va intensificándose mediante la potencialización con otros síntomas. Fue relevante la opresión o apretamiento del tórax anterior, que sumado a sensación de ahogo, conduce a angustia; hay sensaciones térmicas variadas, hormigueo en los brazos, que conducen a percepción de dolor que se irradia a espalda. Es frecuente el uso de diminutivos que denotan subvaloración del síntoma. Los atributos asociados a factores determinantes mostraron como los más importantes la multiplicidad de roles, la ira y el estrés. Como consecuencias el cambio de rol, la angustia y depresión porque "ya no soy la misma". Hay una disyuntiva entre ser indispensable y permitir la sobrecarga de funciones, que involucra diversas formas de afrontamiento en donde los otros se priorizan frente al cuidado de sí mismos.

Conclusiones: los síntomas iniciales son poco definidos, leves y van agudizándose mediante la sumatoria de otros como "una cascada" que se potencializa. Se encontraron trayectorias variadas descritas mediante metáforas y analogías. Un hallazgo novedoso fue el reconocimiento de comportamientos de afrontamiento dirigidos a posponer las propias necesidades y "callar, callar y aguantar'. La espiritualidad emerge como camino para alcanzar un nuevo estado. (c) 2017 Publicado por Elsevier España, S.L.U. en nombre de Sociedad Colombiana de Cardiología y Cirugía Cardiovascular. Este es un artículo Open Access bajo la licencia CC BY-NC-ND (http: / / creativecommons.org/licenses/by-nc-nd/4.0/).
\end{abstract}

\footnotetext{
* Autor para correspondencia

Correo electrónico: frincono@unal.edu.co (F. Rincón-Osorio).
} 


\section{KEYWORDS}

Angina pectoris;

Women;

Qualitative research

\section{Angina symptoms in Colombian women: a meta-synthesis}

\begin{abstract}
Objective: This research responded to the need to understand and analyze the attributes of angina symptoms expressed by Colombian women.

Method: A metasynthetic study of nine research reports developed between 2006 and 2012 . The research was conducted based on the methodology proposed by Noblit and Hare, and Lenz et al.'s Theory of Unpleasant Symptoms.

Results: The attributes of the symptom showed that it starts insidiously and intensifies through potentiation with other symptoms. Chest constriction or tightening was relevant, which, added to the sensation of suffocation, leads to distress. There are varied thermal sensations and tingling of the arms which leads to a pain perception that radiates to the back. The use of diminutives that denote undervaluation of the symptom is frequent. The attributes associated with determining factors showed that the most important ones were multiplicity of roles, anger and stress. Consequences include role change, anguish and depression because "I'm not the same." There is a dilemma between being indispensable and allowing the overload of functions, which involves various forms of coping in which others are prioritized over self-care.

Conclusions: The initial symptoms are poorly defined, mild and worsen with the summation of others in "a cascade" that gains strength. Various paths were found, described by metaphors and analogies. A novel finding was the recognition of coping behaviors aimed at postponing one's own needs and "remaining silent, remaining silent and enduring". Spirituality emerges as a way to reach a new state.

(c) 2017 Published by Elsevier España, S.L.U. on behalf of Sociedad Colombiana de Cardiología y Cirugía Cardiovascular. This is an open access article under the CC BY-NC-ND license (http:// creativecommons.org/licenses/by-nc-nd/4.0/).
\end{abstract}

\section{Introducción}

La problemática de las enfermedades cardiovasculares en la mujer ha venido evidenciándose en las dos últimas décadas de manera clara; según la Organización Mundial de la Salud $(\mathrm{OMS})^{1}$ las tres primeras causas de muerte de mujeres en el mundo son: cardiopatía isquémica, eventos cerebrovasculares e infecciones en las vías respiratorias inferiores. Igual situación se observa en Colombia, en donde, según el Departamento Administrativo Nacional de Estadística (DANE) ${ }^{2}$, para el año 2015 murieron 15.988 mujeres por enfermedad isquémica del corazón (EIC), 7.988 por enfermedades cerebrovasculares y 6.458 por enfermedad de las vías respiratorias inferiores. Además, la tendencia es ascendente en los últimos años como muestra Rincón ${ }^{3}$ en la proyección de mortalidad al 2021 obtenida según datos 2000-2010, al contrario de la presentada en otros países, que han mostrado leve mejoría.

En la actualidad se cuenta con un buen número de estudios que han explorado las diferencias de presentación de la angina, la forma de expresarla y afrontarla según género. Adicionalmente, lo descrito hasta hace unos años se fundamentó principalmente en las manifestaciones de los hombres, llevando a que, por muchos años, las mujeres no identificaran oportunamente sus síntomas y consultaran de manera tardía, así como a que la atención en salud no se diera a tiempo, pues existe desconocimiento sobre las particularidades de esta experiencia en la mujer ${ }^{4,5}$.

Las diferentes formas de presentación y evolución de la enfermedad coronaria entre hombres y mujeres han sido descritas en estudios comparativos, en virtud a las diferencias fisiológicas entre los mismos. El mayor peso de esta diferencia se centra en la prevalencia frecuente de diabetes, hipertensión arterial, obesidad y enfermedades concomitantes en las mujeres. Diversos estudios han permitido señalar que las mujeres con infarto agudo de miocardio (IAM) son unos 10 años mayores que los hombres, llegan a los hospitales una hora más tarde en promedio, desarrollan cuadros clínicos más graves, tienen mayor riesgo de muerte y la intensidad de los tratamientos empleados es proporcionalmente inferior en estas que en los hombres ${ }^{6-10}$. Otros más recientes señalan que la brecha de años entre ambos géneros y su primer evento coronario se ha reducido a 6 años.

En Colombia se han desarrollado estudios que exploran con enfoque cualitativo este tema con el fin de describir desde la perspectiva individual la expresión de los síntomas de angina en la mujer, motivando la necesidad de retomarlos, con el fin de sintetizar y analizar los atributos de los síntomas de angina expresados por la mujer colombiana, a través de la meta-síntesis de nueve investigaciones cualitativas, realizadas en diferentes ciudades colombianas.

Estos estudios han reportado información significativa a través de la cual se desarrolló una síntesis interpretativa, que permitió un aporte a la comprensión de la experiencia del síntoma a través del estudio de las expresiones manifestadas por las mujeres en un proceso cuyo propósito fue responder a la pregunta: ¿Cómo son los atributos del síntoma de angina en la mujer colombiana?

Se consolidó la descripción teórica comprensiva del fenómeno en mención, con el fin de aportar al conocimiento disciplinar de Enfermería y al abordaje que desde la 
interdisciplina contribuye a la cualificación de la atención y el cuidado.

Se fundamentó en la teoría de Lenz et al., cuya estructura contiene tres constructos:

- Dimensiones del síntoma.

- Factores determinantes.

- Consecuencias de este.

\section{Materiales y métodos}

Se realizó una búsqueda detallada en bases de datos (EBSCO, BVS, MedLine, PubMed, CINHAL) y en el repositorio de la Biblioteca de la Universidad Nacional de Colombia, de estudios cualitativos sobre la experiencia de los síntomas de angina en la mujer, circunscrita a la participación de informantes de nacionalidad colombiana. De esta búsqueda se encontraron 11 estudios, que cumplían con los criterios de inclusión. Para la verificación de la calidad de los estudios se utilizaron los criterios propuestos en el Critical Appraisal Skills Programme Español (CASPe) para la evaluación de estudios cualitativos ${ }^{11}$. A partir de esta verificación se descartaron dos estudios debido a la falta de claridad sobre el procedimiento y el análisis de los datos obtenidos. Se consideró la metasíntesis como la mejor forma de ampliar la comprensión del fenómeno en estudio, en razón a que esta metodología permite la estructuración sistemática de las expresiones y los elementos circundantes a la experiencia, en este caso, de los síntomas de angina en la mujer en Colombia. La investigación se condujo con base en la propuesta metodológica de Noblit y $\mathrm{Hare}^{12}$ para la realización de metasíntesis, tal como se observa en la tabla 1.

Los hallazgos en general están referidos a 142 mujeres participantes. El análisis detallado y exhaustivo de la información identifica una serie de categorías que aportan a la interpretación y comprensión de la experiencia de las participantes en relación con los síntomas y su significado y además identifica nuevos elementos de la experiencia que contribuyen al desarrollo teórico, base para generar nuevas opciones de cuidado ${ }^{13-21}$.

\section{Consideraciones éticas}

Se contó con el aval del Comité de Ética de la Facultad de Enfermería de la Universidad Nacional de Colombia y de cada una de las instituciones donde se desarrollaron los estudios. (Resolución No. 077 del 2006 del Consejo de Facultad de Enfermería).

\section{Resultados}

\section{Síntomas: atributos relevantes}

El tema más frecuente y notable fue la opresión (más que dolor) en el tórax anterior, pero también identificó una secuencia precedente: sensaciones térmicas y hormigueo distal en miembro superior derecho o izquierdo que se dirige luego al tórax en donde se suma con nuevas sensaciones de calor, disnea, opresión, tanto en el tórax como en el cuello (denominada atoramiento, atragantamiento) las cuales
Tabla 1 Método metaetnografía de Noblit y Hare (1988)

Fase 1. Iniciando el
proceso
Fase 2 . Decidiendo lo
que es relevante

En esta etapa se establece el área de interés y la necesidad de la metasíntesis

Fase 3. Lectura de los En esta fase se realiza lectura y artículos relectura de los artículos, con el fin de encontrar las metáforas interpretativas en cada uno.

Fase 4. Determinación de cómo los estudios En esta fase los estudios son puestos juntos para buscar las relaciones entre sí, mediante la están relacionados comparación recíproca y por refutación.

Fase 5. Trasladar los Las metáforas y los temas son estudios uno dentro comparados en tanto que se de otro conservan las metáforas centrales intactas

Fase 6. Sintetizando las traslaciones

En esta fase el investigador sintetiza las partes para crear el todo

Fase 7. Expresando la Se escribe la síntesis. síntesis

Tomada literalmente de: COFFMAN, Maren. Cultural Caring in Nursing practice: a Meta-synthesis of qualitative research. J Cultural Diversity. p.101.

acumuladas disparan la opresión profunda en todo el tórax, o el dolor y la angustia, que define la solicitud de ayuda o la búsqueda de un alivio. Esto fue identificado con la categoría "cascada de síntomas" y "el pequeño gigante", por la progresión de los síntomas hasta determinar algo no controlable.

La irradiación más frecuente fue escápula izquierda y cuello. Las analogías son frecuentes y la integración con los eventos de la vida cotidiana son el marco de la narrativa. Se encontraron subgrupos de mujeres especialmente en la zona andina central y sur, que describieron sus síntomas como algo pasajero y sin importancia, subestimando la sensación y por lo tanto ignorándola: "es una molestia", "es una bobadita', dándole variadas interpretaciones, posponiendo permanentemente su atención y priorizando la atención al otro: familia, trabajo, etc.

Dentro de las interpretaciones de las mujeres sobre sus síntomas, se identifican hipótesis asociadas a problemas gastrointestinales (gases, gastritis, intolerancia a las comidas y úlcera), y cambios en la temperatura ambiente. De igual forma, su narrativa se orienta hacia esas posibilidades que son ajenas a la enfermedad coronaria, pues en su imaginario ésta no se encuentra contemplada, lo que lleva a la confusión que dilata la consulta y deriva en la utilización de estrategias alternativas y a la automedicación.

Esta situación cambia en algunos casos en los que la mujer tiene historia familiar de enfermedad coronaria, ya que reconoce el riesgo y por tanto presta más atención a los síntomas lo cual la motiva para hacer una consulta más temprana. 


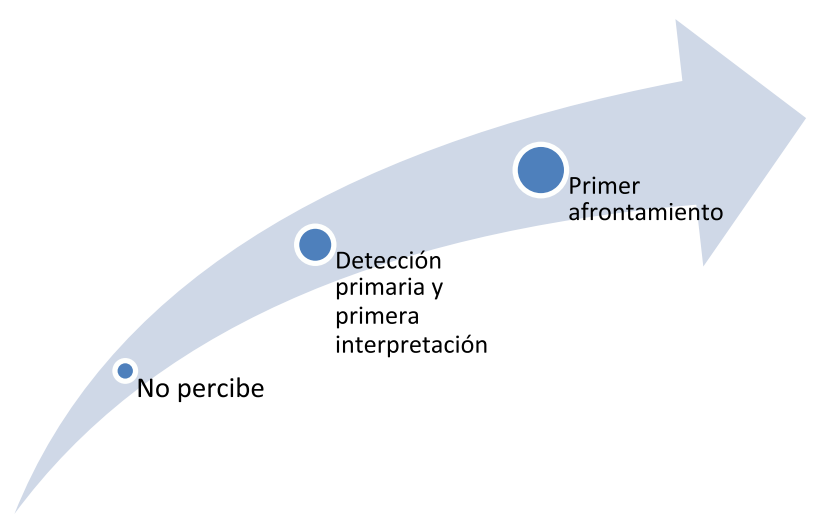

Figura 1 Proceso interpretativo.

La integración de los síntomas presenta procesos en cuanto a:

- Direccionalidad: marca trayectorias, es decir, áreas corporales de transmisión.

- Consolidación del síntoma: inicio con un solo síntoma que va intensificándose en sí mismo y en compañía de otros hasta comprometer la funcionalidad, momento en el cual se decide pedir ayuda.

- Proceso interpretativo que define la respuesta (fig. 1).

A su vez, el primer afrontamiento generalmente se caracteriza por sopesar, contrastar y subvalorar los síntomas, lo que conduce a posponer el cuidado o pedir ayuda.

Se observa que al inicio se expresan los síntomas llamados acompañantes, marcando una señal que frecuentemente se desatiende, para luego, consolidarse a través de su propia intensificación y la potencialización con otros; un solo síntoma no logra esclarecer la necesidad de ayuda pero sí la sumatoria de varios.

\section{Factores determinantes de la presentación del síntoma ${ }^{22}$}

Se identificó un proceso cognitivo sobre lo que la mujer sabe, como base de las interpretaciones que desarrolla para comprender lo que está sucediendo. A su vez este contenido incluye desde el conocimiento oído que se repite, hasta el que se asume como una verdad y se comprende (fig. 2).

Ese conocimiento hace referencia a dos aspectos: la presencia de la enfermedad y los asuntos que se relacionaron con la aparición del síntoma. Los contenidos relevantes se refirieron a:

- Los denominados factores de riesgo comúnmente reconocidos y derivados del conocimiento en salud, destacando el estrés.

- Los aspectos que ellas han autodiagnosticado como los que enferman: disgustos, rabia y sufrimiento, que llevan igualmente al estrés.

- El peso del diagnóstico asignado por otros.

El nivel de conocimiento expresado a través de lo que les han dicho, refleja diferentes niveles de comprensión sobre lo que sucede, generalmente expresados con analogías, pero tendiente a dejar de lado la comprensión del fenómeno anormal, para revisar las causas que llevaron a éste. Los exámenes diagnósticos realizados y por hacer, hacían parte del arsenal de información que ayudaba a tener la sensación de control y seguridad en la situación.

Se advierte en este proceso un traslado del poder a quien posee el conocimiento (funcionario de salud), momento en el que se exalta tanto el diagnóstico por quien lo dijo (connotación de verdad), como la importancia de la situación por el impacto que genera (gravedad de la enfermedad). Esto determina la percepción de pérdida del control sobre los asuntos que influyen en su salud y por lo tanto genera una actitud pasiva y dependiente. Se refieren a la enfermedad como un asunto externo, del cual hablan de manera impersonal y cuyo control lo tiene otro (fig. 2: locus de control externo). Como resultado, el proceso cognitivo se debilita y el conocimiento útil para respaldar acciones en pro de la propia salud, no se integra completamente en la estructura mental de la mujer, derivando en una detallada descripción de las circunstancias asociadas al evento, rica en asociaciones pero sorda a la voz de su cuerpo. Siempre hay "alguien" al lado con el que la mujer está interactuando que determina positiva o negativamente su decisión sobre sí misma.

Sobre su comprensión de la enfermedad se identificaron varias expresiones inexactas sobre el corazón. Los términos "yo pienso" y "tengo entendido", se asociaron con la incertidumbre y el desconocimiento frente al origen de sus síntomas, persistentemente enfocados a una manifestación física en el pecho "porque todo lo que queda aquí (se toca el hemitórax izquierdo) es del corazón'”.

Dentro de los factores determinantes fue clara la idea de la mayoría en cuanto a los factores de riesgo cardiovascular (FRCV) como causales de la enfermedad, especialmente aquellos que se relacionan con sus hábitos y forma de vida: alimentación inadecuada, cigarrillo, alcohol, diabetes, etc.

Se destacó el estrés asociado a los múltiples roles: madre, esposa, cuidadora de adultos mayores, niños o personas con discapacidades. Hubo referencias especiales, disfunción de relaciones de pareja y duelos por muerte o separación. La herencia y la edad tuvieron referencias específicas por considerarse factores inamovibles similares a una sentencia.

Se evidencia que diferencian los factores reconocidos como generadores de la enfermedad y los desencadenantes de los síntomas; dentro de estos últimos el estrés es

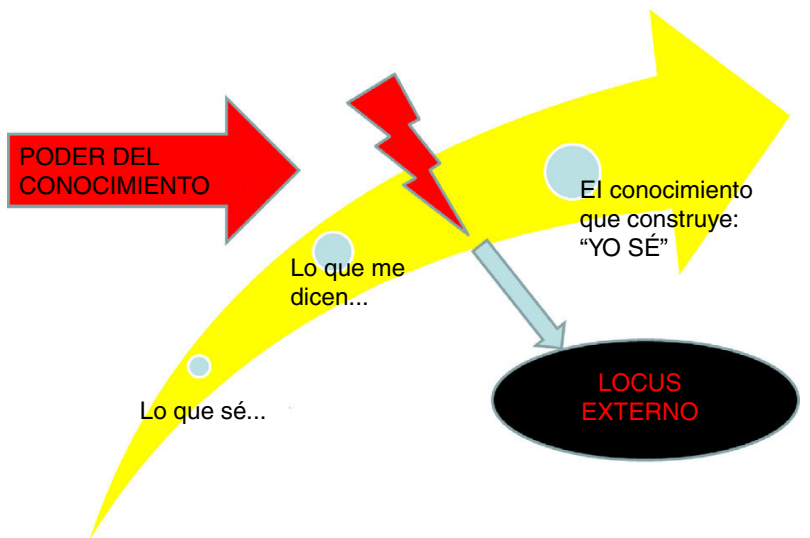

Figura 2 Proceso cognitivo sobre la propia salud. 


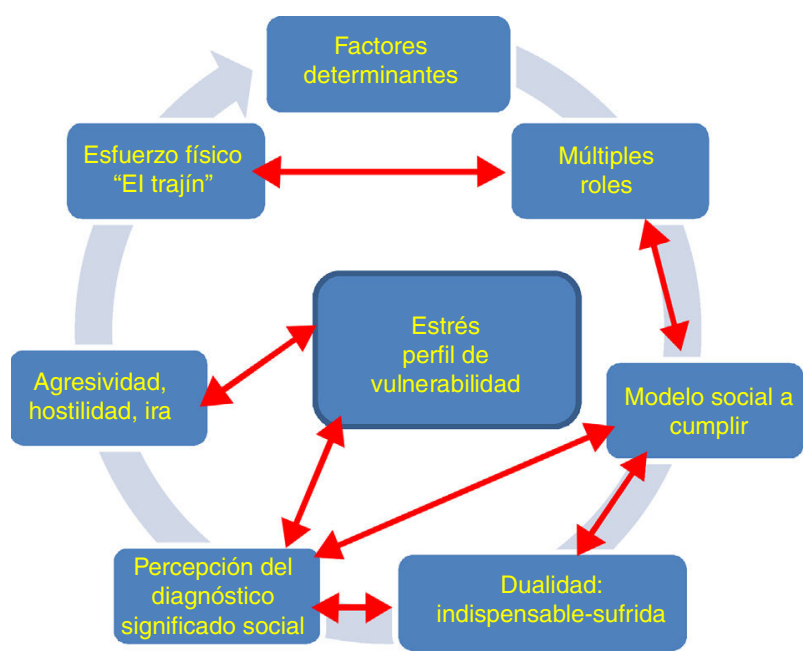

Figura 3 Factores determinantes de los síntomas y la enfermedad.

percibido como el mayor y menos frecuente la actividad física, pero no la intensa, sino aquella relacionada con "el trajín" (fig. 3).

El estrés es considerado producto de las relaciones y compromisos generados en el marco de los diferentes roles, claramente relacionados con valores y creencias que definen lo que se espera que sea y haga la mujer. Un ejemplo de ello es sentirse responsable de todo aquello que le rodea, especialmente si se relaciona con la familia: salud, situación económica, estabilidad laboral, situaciones emocionales, pérdida y duelo, algunas de ellas asociadas a desplazamiento y violencia, desesperanza y responsabilidad excesiva.

La sumatoria de situaciones demandantes, múltiples funciones y presencia de duelos van determinando la conformación de una identidad de "mujer sufrida".

Cuidar a otros, ancianos, niños y enfermos en situaciones de cronicidad, se asoció por parte de las mujeres con la presentación de los síntomas y escenario de enfermedad. Esta función relacionada con el género femenino desde sus orígenes antropológicos, sumada a otras responsabilidades tradicionales y emergentes, ha demostrado potencializar el factor de riesgo estrés, lo cual se expresa por un comportamiento reactivo ante estímulos cotidianos y dificultad en las relaciones interpersonales para la búsqueda de apoyo y ayuda. Barrera et al. ${ }^{23}$ señalan que la mujer, además de convivir con la enfermedad crónica, frecuentemente es cuidadora de otros aquejados por estas enfermedades, lo cual aporta a la construcción de un perfil de vulnerabilidad.

De igual forma, las relaciones de pareja se asocian a estrés en condiciones de disfunción, tal como lo señala Faur $^{24}$ quien refiere el estudio llevado a cabo en la Universidad de Ohio en el que demostraron que las mujeres tenían mayor sensibilidad frente a las interacciones negativas que los hombres y que tenían recuerdos más vívidos y detallados de las discusiones que sus maridos, mostrando cambios hormonales sostenidos hasta 24 horas posteriores a la discusión.

En cuanto a la actividad física como factor desencadenante del síntoma tiene un contenido adicional que está asociado a las tareas del hogar y es denominado "el trajín" que involucra no solo las actividades diarias sino que se complejiza con la carga emocional que está asociada al rol de mujer sin apoyo social.

Las ideas expresadas por las mujeres en relación con las circunstancias en las que aparecen los síntomas, no se mencionan directamente sino que se desarrollan mediante la narrativa detallada en donde se identifican relaciones, inconformidades y funciones, muchas de ellas con carácter de indispensable y por tanto obligatorio en su cumplimiento. Se expresa como dualidad de sentimientos: percepción de carga y sufrimiento versus sentirse indispensable. Señalan que la acumulación de las funciones percibidas como cargas van generando progresivamente rabia o ira para el manejo de los asuntos familiares y en algunos casos laborales, dejando el sentimiento de inconformidad. El sufrimiento se hace evidente en sus expresiones relacionadas con pérdidas ya sea de seres queridos, económicas, de relaciones, etc. que consideran las afectó y las condujo a la presentación de los síntomas.

El peso del diagnóstico médico tiene una influencia significativa en el comportamiento de las mujeres lo que se observó en las narrativas, ya sea como sentencia o como mensaje con la capacidad de dotar de importancia social su situación. Los términos utilizados para describir la situación de la paciente influyen significativamente en la percepción de autoeficacia y por lo tanto en la motivación para el cambio, pues el lenguaje utilizado ininteligible genera incertidumbre y despersonalización.

Cuando la referencia médica cobija a la familia como factor de riesgo familiar, se suma la importancia del criterio de una persona externa, socialmente reconocida (médico) y los resultados de los exámenes diagnósticos definiendo la enfermedad como una sentencia inmodificable. Se deriva así, una interpretación en la que la mujer no es la responsable de su situación de salud, sino que es un estado que debe aceptar. Esto conducirá a comportamientos de afrontamiento caracterizados por la aceptación pero con poca capacidad de control.

Diferente situación se observó en algunos casos en los que el evento cardíaco sustrajo de la invisibilidad a la mujer y le imprimió una importancia inesperada frente a la familia, como oportunidad de ser reconocida como sujeto de cuidado y no solamente cuidadora.

Las mujeres asocian las preocupaciones tanto con la generación de la enfermedad como con el factor desencadenante de los síntomas. Esta preocupación es implícitamente asociada con "la pensadera", es decir, el recabar insistentemente en un problema generalmente familiar sobre el cual no tiene poder de resolución y que de manera permanente determina un estado de estrés crónico. Ellas mismas se identifican como mujeres nerviosas, aceleradas, sensibles, sentimentales y preocupadas por todo lo que pasa en su hogar, lo que les afecta directamente.

Este tema ha sido ampliamente documentado en la literatura y se ha identificado el denominado patrón de conducta tipo A (PCTA) que se caracteriza por la sensación permanente de urgencia por el tiempo, irritabilidad, ambición y competitividad, expresión intensa y que refleja una situación en que la persona se encuentra agresivamente involucrada en una lucha incesante, para lograr eficiencia. La hostilidad dentro del PCTA constituye un constructo que apoya al proceso predictivo de enfermedad cardiovascular e incluye tres componentes: la ira como componente afectivo 
o emocional; la hostilidad como componente cognitivo y la agresión como componente conductual ${ }^{25}$.

Estos componentes se encuentran permanentemente relacionados y han demostrado ser factores de riesgo cardiovascular, en especial para EC, constituyendo el llamado síndrome $\mathrm{AHI}$ (agresión, hostilidad, ira).

Algunas de las causas referidas en las narrativas fueron: la priorización de sus obligaciones, su creencia acerca de que las mujeres se mueren por otras causas, la no identificación de los síntomas, además de experiencias previas con los servicios de salud y el principio de que deben ser fuertes.

\section{Consecuencias de los síntomas y afrontamiento de la enfermedad ${ }^{26}$}

A lo largo de las entrevistas y de manera espontánea, las participantes expresaron sentimientos y formas de afrontamiento de los síntomas, dentro de las cuales se destacaron las que señala la figura 4.

La enfermedad y sus síntomas dan lugar a cambios determinantes en la trayectoria de vida de las mujeres, con diferentes etapas que inician con la ansiedad, la incertidumbre y una gran preocupación constante sobre el cumplimiento de sus roles, porque no los puede ejercer por su estado o por orden médica o por protección de su familia. Esto derivó dos situaciones: en la mayoría ocasionó sentimientos de minusvalía y tristeza y en algunos pocos casos, una oportunidad de recibir cuidados y reconocimiento de la familia. Prácticamente en todas las participantes estuvo presente la idea de la religiosidad o de la espiritualidad, esencial apoyo para continuar la recuperación y adaptación (fig. 4).

\section{Discusión}

A lo largo del análisis de las narrativas, fue relevante el hecho de que los primeros síntomas, leves, difusos y variables, usualmente eran más de cinco síntomas simultáneos que describían trayectorias.

Algunos de ellos no fueron percibidos por una buena parte de las mujeres, fenómeno que puede ser interpretado a través del concepto de "sobreadaptación" que refiere Tajer"27, quien explica que es una modalidad de respuesta a situaciones de cambios (migraciones, desplazamientos, cambios socioeconómicos súbitos familiares, etc.) en donde la exigencia de adaptación rápida a otro estilo de vida les exige una "modalidad de funcionamiento psíquico operativo, con

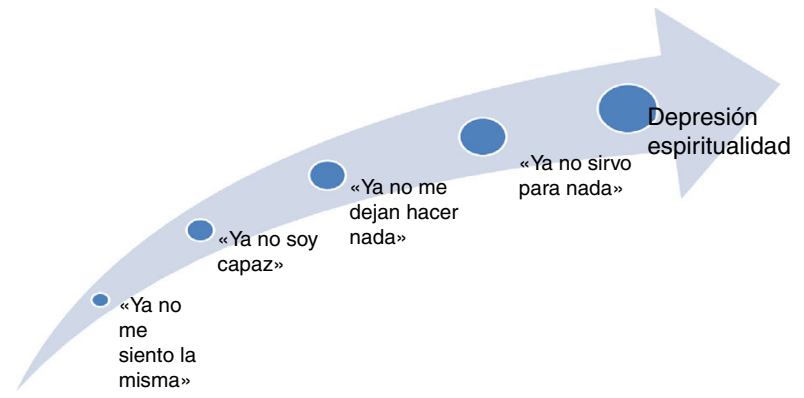

Figura 4 Consecuencias de los síntomas y la enfermedad. tendencia al sobreesfuerzo y con dificultades para interpretar y privilegiar las propias necesidades que no estén ligadas a la búsqueda de ascenso social". En este marco de ideas ocurre lo que ha sido denominado la "externalización", es decir, el esfuerzo de la persona por responder de manera eficiente a todos los estímulos y exigencias del medio dejando de lado la percepción de su mismo cuerpo, sorda a sí misma. Fue bien descrito desde la voz de las mujeres: "No le hago cama al dolor", o "la cultura del aguante".

Otra explicación frente a este fenómeno se deriva de los estudios de Guilligan ${ }^{28}$, psicóloga y filósofa estadounidense, quien estudió los procesos de formación de la moral en niñas. Denominó la primera etapa "el sí mismo", en donde la tendencia es hacia el cuidado esencial de sí y mediante la vivencia de diferentes experiencias se hace una transición hacia lo social moral convencional por considerarse la primera como egoísta. Entra entonces a establecer la relación con otros emergiendo el concepto de responsabilidad y pospone la atención en sí misma para realzar la importancia del otro. Posteriormente sigue la etapa post convencional en la que la mujer tiene consolidados los criterios para la toma de decisiones dentro de los cuales aparece en una posición preponderante: el otro con un criterio de priorización basado en quien esté más necesitado, constituyéndose en el principal criterio para la toma de decisiones; emerge aquí el concepto de ética del cuidado, en el sentido de priorizar la función de cada una en el cuidado del otro lo cual incluye el yo y los otros en la responsabilidad del cuidado.

En estas condiciones, la angustia y la ansiedad son comunes, pero derivadas de su preocupación en cuanto al cumplimiento de funciones.

Como resumen de lo anterior se plantea la siguiente hipótesis teórica: la tendencia del proceso es a la creación de trayectorias sintomáticas a través de las variables de tiempo e intensidad: un inicio en donde se expresan síntomas subyacentes (escondido, disimulado) de intensidad leve a moderada, que luego se van potencializando manifestándose en un cuadro más concreto concentrado en tres síntomas: opresión, angustia y dolor, que la paciente sí puede identificar y calificar, para luego continuar en una forma descendente dejando la sensación de maltrato, malestar y a veces una percepción de sensaciones casi imperceptibles. En esta fase, el síntoma pierde importancia en la percepción de la mujer y por tanto no genera la búsqueda de ayuda.

Respecto a las consecuencias generadas al percibir los síntomas, fue claro el tema que reconoce el cambio descrito de manera melancólica y tendiente a la depresión: "Ya no soy la misma". Se asume la vida en un proceso de decadencia que no permite avizorar la capacidad de autocuidado y rehabilitación. Toma un lugar preponderante el miedo a la incapacidad y se reconoce la necesidad de cambiar por lo que se plantean las promesas desde la perspectiva teórica. También hay un subgrupo que encontró en la enfermedad un punto de apoyo para ser reconocida en familia y en sociedad frente a una larga trayectoria vital ajena a sus expectativas. Ser admirada por "ser muy fuerte" y tolerar los dolores dio cierto ánimo a algunas mujeres, pero reforzó el carácter de que "aguantar"' es una cualidad en el ser humano.

Todas estas formas de interpretar las sensaciones en el marco de la trayectoria de vida permitieron explicar la interpretación de los síntomas que lleva a un afrontamiento 
específico en un contexto definido por la trayectoria de vida: interpretar, subvalorar, posponer, reinterpretar, experimentar confusión e incertidumbre, dar valor al síntoma, pedir ayuda, son formas de responder, que atañen a una forma de pensar y vivir.

La forma de afrontamiento puede tipificarse a lo largo de la experiencia de salud y enfermedad: inicia en el afrontamiento de los factores de riesgo y en los comportamientos de autocuidado o su ausencia, por lo que se le considera de especial interés. Según algunos autores ${ }^{29}$ "se entiende el afrontamiento como aquellos esfuerzos cognitivos y conductuales constantemente cambiantes que se desarrollan para manejar las demandas específicas externas y/o internas que son evaluadas como excedentes o desbordantes de los recursos del individuo"'. Se observan tipos de afrontamiento como pedir ayuda profesional, que en la mayoría de los casos estaba mediada por una persona, generalmente familiar, que la conduce o la induce a buscarla, compartir la experiencia con legos, aplicarse medidas paliativas caseras como: sobarse, tomar pastillas, tomar agua, etc. Buscar formas relacionadas con la actividad para aliviar el síntoma: suspender la actividad, cambiar la posición.

Influyen en el afrontamiento sentimientos asociados a la desprotección de seres queridos, la preocupación por no molestar a otros, el miedo a la muerte y la incapacidad y el concepto propio de la enfermedad.

La categoría: "Señor, en tus manos estoy" muestra cómo la forma de afrontamiento de los síntomas de angina en las mujeres con frecuencia se encuentra impregnada de creencias y valores religiosos que constituyen un poderoso soporte en el momento crítico.

La valoración cognitiva (percepción de amenaza o incertidumbre) influye no sólo en las estrategias de afrontamiento, sino también en los resultados adaptativos como la tensión emocional, la ansiedad, la autoestima o la percepción de control sobre la enfermedad "Aguantar, callar y callar" categoría encontrada en el estudio de Narváez ${ }^{18}$ es a su vez un factor determinante de la enfermedad coronaria, como una forma de afrontar la enfermedad y de vivir con ella. Más allá, define cómo aborda las consecuencias de esta: la incapacidad, el cambio de rol, las alteraciones cognitivas, las funcionales: un patrón de comportamiento a esta medida requiere de un análisis y de estrategias diferentes para trabajar la prevención primaria, secundaria y terciaria.

\section{Conclusiones}

Se identificaron los principales atributos de los síntomas de angina en la mujer, dentro de los cuales fueron relevantes la opresión precordial o torácica que podía extenderse al cuello, sensación de ahogo, ansiedad que al sumarse, desencadenaban el síntoma dolor, cuya irradiación era predominantemente hacia la espalda y en algunas ocasiones a la mandíbula.

Se identificaron formas de comprensión y vivencias de la enfermedad coronaria que revelan una persistente tendencia a la subvaloración de los síntomas y a posponer el propio cuidado por atender las necesidades de otros. Además se pudieron identificar elementos específicos que las mujeres asocian con los factores que determinan sus síntomas, las características de estos, más la variabilidad en su trayectoria, y las consecuencias que tienen en su vida.

Se destaca el hallazgo adicional sobre el afrontamiento de los síntomas que da importantes herramientas para el cuidado. Está presente en todo el proceso y se conjuga con el proceso cognitivo para configurar las respuestas de las mujeres frente al síntoma.

Se concluye que la expresión de la experiencia de los síntomas de angina en la mujer colombiana, está matizada por la cultura, especialmente en lo que significa el ser mujer al interior de su hogar y lo que se espera de ella, de la misma manera en la forma en que ésta influencia la descripción de la intensidad y compromiso del síntoma.

Finalmente ha emergido el concepto de vulnerabilidad para abarcar todas las anteriores reflexiones y dar lugar a nuevos hallazgos bajo esta conceptualización pertinente a los resultados encontrados.

\section{Financiación}

Esta investigación fue financiada por la Facultad de Enfermería de la Universidad Nacional de Colombia mediante Convocatoria UGI-2010, Unidad de Gestión e Investigación. Código Hermes 12966.

\section{Conflicto de intereses}

Ninguno.

\section{Bibliografía}

1. Organización Mundial de la Salud. Centro de Prensa [internet]. Salud de la mujer. Nota descriptiva No. 334 Septiembre de 2013. [Acceso 17 feb 2017]. Disponible en: http://www. who.int/mediacentre/factsheets/fs334/es/.

2. Departamento Nacional de Estadística [internet]. Estadísticas vitales. [Acceso 19 feb de 2017]. Disponible en: https://www.dane.gov.co/index.php/estadisticas-por-tema/ salud/nacimientos-y-defunciones/defunciones-no-fetales/ defunciones-no-fetales-2015.

3. Rincón F. La enfermedad coronaria en la mujer: un asunto de cuidado. Primera edición Bogotá: Editorial Universidad Nacional de Colombia; 2013.

4. Laxmi S Mehta. et al. Acute Myocardial Infarction in Women A Scientific Statement From the American Heart Association. Circulación [internet]. [Acceso 19 feb 2017]. Disponible en: http: / /circ.ahajournals.org/content/early/2016/01/25/CIR. 0000000000000351.

5. De Von H, Zerwic J. The symptoms of instable angina: Do women and men differ? Nursing Research. 2003;52:108-18.

6. Nguyen HL, Gore JM, Saczynski JS, Yarzebski J, Reed G, Spencer FA, et al. Age and sex differences and 20-year trends (1986 to 2005) in prehospital delay in patients hospitalized with acute myocardial infarction. Circ Cardiovasc Qual Outcomes. 2010;3:590-8.

7. Caldwell M, Miaskowski C. The symptom experience of angina in women. Pain management nursing. 2000;1:69-78.

8. Rosenfeld AG, Knight E, Steffen A, Burke L, Daya M, DeVon $\mathrm{H}$. Symptom clusters in patients presenting to the emergency department with possible acute coronary syndrome differ by sex, age, and discharge diagnosis. Heart Lung. 2015;44:368-75. 
9. Devon H. Sensitivity, specificity, and sex differences in symptoms reported on the 13-item acute coronary syndrome checklist. J Am Heart Assoc. 2014;3:e000586.

10. Horne R, James D, Petrie K, Weinman J, Vincent R. Patients interpretation of symptoms as a cause of delay in reaching hospital during acute myocardial infarction. Heart. 2000;83:388-93.

11. Programa de habilidades en lectura crítica español [internet]. Plantilla para estudios cualitativos. [Acceso 27 feb 2016]. Disponible en: http://www.redcaspe.org/ system $/ \mathrm{tdf} /$ materiales/plantilla_cualitativa.pdf?file=1\&type= node\&id $=147 \&$ force $=$.

12. Noblit GW, Hare RD. Meta-ethnography: synthesizing qualitative studies. Sage: Newbury Park; 1988

13. Cortés LA. Tipificación del síntoma dolor torácico tipo isquémico en la mujer, a la luz de la teoría de los síntomas desagradables. Tesis de Maestría. Bogotá: Universidad Nacional de Colombia; 2006.

14. Millan EM. Experiencia del dolor torácico en la mujer con infarto del miocardio. Tesis de Maestría. Bogotá: Universidad Nacional de Colombia; 2008.

15. Soto AM. Factores que intervienen en la demora de la solicitud de atención médica o de enfermería en mujeres que presentan dolor torácico de origen coronario. Tesis de Maestría. Bogotá: Universidad Nacional de Colombia; 2006.

16. Campo E. Caracterización del dolor torácico tipo isquémico en un grupo de mujeres a la luz de la teoría de síntomas desagradables. Tesis de Maestría. Bogotá: Universidad Nacional de Colombia; 2007.

17. Medrano MP. Caracterización de los síntomas de angina en mujeres hospitalizadas en la Clínica San Juan de Dios de Cartagena. Tesis de Maestría. Bogotá: Universidad Nacional de Colombia; 2012.

18. Narváez CO. Caracterización de los síntomas de angina de pecho en un grupo de mujeres del departamento de Nariño en el marco de la teoría de los síntomas desagradables de Lenz y Cols. San Juan de Pasto: Universidad Nacional de Colombia, Estudio Multicéntrico; 2009.
19. Romero E. Caracterización de los síntomas de angina de pecho en mujeres a la luz de la teoría de síntomas desagradables Clínica Cartagena del Mar. Tesis de Maestría. Bogotá: Universidad Nacional de Colombia; 2007.

20. Moreno Z. Diferencia en los síntomas de angina que presentan hombres y mujeres atendidos en la Fundación Cardiovascular de Colombia Tesis de Maestría. Bogotá: Universidad Nacional de Colombia; 2009.

21. Rincón F. Caracterización de los síntomas de angina en un grupo de mujeres adultas con cateterismo positivo para enfermedad coronaria, en el marco de referencia de la teoría de los síntomas desagradables de Lenz y Cols. Tesis de Maestría. Bogotá: Universidad Nacional de Colombia; 2007.

22. Lenz ER, Pugh LC, Milligan RA, Gift A, Suppe F. The middle-range theory of unpleasant symptoms: An update. Advances in Nursing Science. 1997;19:14-27.

23. Barrera L, Pinto N, Sánchez B. Caracterización de los cuidadores familiares en América Latina. 2010. En: Barrera L, Chaparro L, Carrillo M, Pinto N, Sánchez B, editores. Cuidando a los cuidadores familiares de personas con enfermedad crónica. Bogotá: Universidad Nacional de Colombia; 2010. p. 45-55.

24. Faur P. Corazón y Emoción. 4a Jornada de Psicocardiología. Buenos Aires. 1 $\stackrel{a}{a}$ edición Instituto de Psicocardiología; 2010.

25. Lacovella J, Troglia M. La hostilidad y su relación con los trastornos cardiovasculares. Psico-USF. 2003;8:53-61.

26. Lenz ER, Suppe F, Gift AG, Pugh LC, Milligan RA. Collaborative development of middle-range nursing theories: Toward a theory of unpleasant symptoms. Advances in Nursing Science. 1995; 17:1-13.

27. Tajer D. Heridos corazones. Subjetividad y vulnerabilidad coronaria en varones y en mujeres. Paidós: Buenos Aires; 2009.

28. Gilligan C. In a different voice. Psychological theory and women development. Estados Unidos: Harvard University Press; 2003.

29. Castaño EF, León Del Bargo B. Estrategias de afrontamiento del estrés y estilos de conducta interpersonal. Int J Psychol Psychological Therapy. 2010;10:245-57. 